\title{
Structure of a Langmuir Film on a Liquid Metal Surface
}

\author{
H. Kraack, ${ }^{1}$ B. M. Ocko, ${ }^{2}$ P. S. Pershan, ${ }^{3}$ E. Sloutskin, ${ }^{1}$ \\ M. Deutsch ${ }^{1 *}$
}

\begin{abstract}
The structure of organic monolayers on liquid surfaces depends sensitively on the details of the molecular interactions. The structure of a stearic acid film on a mercury surface was measured as a function of coverage with angstrom resolution. Unlike monolayers on water, the molecules were found here to undergo a transition from surface-parallel to surface-normal orientation with increasing coverage. At high coverage, two condensed hexatic phases of standing-up molecules were found. At low coverage, a two-dimensional (2D) gas phase and condensed single- and double-layered phases of flat-lying molecular dimers were revealed, exhibiting a 1D longitudinal positional order. This system should provide a broader tunability range for nanostructure construction than solid-supported self-assembled monolayers.
\end{abstract}

Amphiphilic molecules like alcohols and fatty acids consist of a hydrophilic head and hydrophobic tail. When placed on the surface of an aqueous bulk, such molecules, surface-confined by the head group-surface hydrophilic attraction, often form a monolayer, known as a Langmuir monolayer (LM) $(1,2)$. Because of their quasi-2D nature and the molecular alignment by the surface, the structure and properties of LMs depend sensitively on the details of the architecture of the constituent molecules and their inter- and intramolecular interactions. Moreover, this structure can be varied easily by compression, temperature variation, or adsorption of solutes from the subphase. Thus, molecular-level studies of the structure of LMs are invaluable for elucidating the nature of interand intramolecular interactions in organic, organometallic, and biologically active molecules. Having intrigued researchers since 1891 (3), LMs have also been studied as models of 2D matter, of cell membranes, and as a route to nanoengineering and molecular electronics $(1$, $2,4)$. Their angstrom-scale structure was first determined only in 1987, by in situ synchrotron x-ray methods (5-7). Since then, many such studies have revealed a wealth of structures, phases, and transitions in LMs of chain molecules on various aqueous subphases $(1,2,8)$. Invariably, the combination of van der Waals attraction between the alkyl chains and the hydrophobic repulsion between the chains and the aqueous subphase drives the alignment of the molecules' long axis at, or close to, the surface normal, regardless of their in-plane organization.

1Physics Department, Bar-Ilan University, Ramat Gan 52900, Israel. ${ }^{2}$ Physics Department, Brookhaven National Laboratory, Upton, NY 11973, USA. ${ }^{3}$ Physics Department and Division of Applied Sciences, Harvard University, Cambridge, MA 02138, USA.

*To whom correspondence should be addressed. Email: deutsch@mail.biu.ac.il
Here, we report angstrom-resolution x-ray measurements of the coverage dependence of the structure of a LM on a liquid $\mathrm{Hg}$ subphase. The use of a nonaqueous, yet liquid, Hg subphase causes a drastic change in the chain-subphase interaction, from a strong hydrophobic repulsion for the aqueous subphases used to date to a strong attraction for the $\mathrm{Hg}$ surface. This results in the formation of an intriguing phase sequence, in which the orientation of the molecules with respect to the surface changes from parallel to normal. The high-coverage phases are similar to those observed on aqueous subphases, with surface-normal-oriented molecules (8). The low-coverage phases, however, consist of single or double layers of flat-lying molecular dimers, the x-ray diffraction patterns of which suggest a 1D smectic-like longitudinal positional order. Such phases have not been reported for aqueous subphases. A coexistence range between the condensed flat-lying and standing-up phases is also found.

Thermodynamic and kinetic data are available for organics on $\mathrm{Hg}(9)$, but only three structural $\mathrm{x}$-ray studies have been published (10-12). They reported close-packed phases with surface-normal molecules, as a result of the high coverages used. Here we use stearic acid $\left[\mathrm{CH}_{3}-\left(\mathrm{CH}_{2}\right)_{16}-\mathrm{COOH}\right]$, for which the estimated carboxyl head group- $\mathrm{Hg}$ subphase bond strength (10), $\sim 1.5 \mathrm{~kJ} / \mathrm{mol}$, is even lower than the carboxyl-water hydrogen bond strength (13), $\leq 10 \mathrm{~kJ} / \mathrm{mol}$. Stearic acid is expected, therefore, to form a $\mathrm{LM}$ on $\mathrm{Hg}$, where the molecules should have high surface mobility and thermal equilibrium structures should be obtained at any given coverage. This expectation is confirmed by $\mathrm{x}$-ray reflectivity $(\mathrm{XR})(14,15)$, grazing incidence diffraction (GID) (14-17) (measuring the structure normal and parallel to the surface, respectively), Bragg rod (BR) (measuring the molecular tilt's magnitude and direction)
(14-17), and surface-pressure $(\pi) /$ moleculararea $(A)$ isotherm $(9,18)$ measurements.

Our measured isotherm on $\mathrm{Hg}$ (circles) and that measured on water (18) (line) are shown in Fig. 1. The much larger values of $A$ and the associated $\pi$ on $\mathrm{Hg}$ stand out clearly. The isotherm's fast-increasing region at $110 \leq A \leq 200 \AA^{2} /$ molecule is well fitted by the 2D "ideal" gas law (dashed line), $\pi(A-$ $\left.A_{0}\right)=k_{\mathrm{B}} T$, where $k_{\mathrm{B}}$ is the Boltzmann constant and $A_{0}$ is the exclusion area due to the finite molecular size. The fitted $A_{0}=(112 \pm$ 10) $\AA^{2} /$ molecule is very close to the calculated $24.4 \AA \times 4.85 \AA \approx 118 \AA^{2}$ area of a molecule lying parallel to the surface (19). When $A$ decreases below $110 \AA^{2} /$ molecule, three distinct $\pi$-regions are observed: a slow rise, a plateau, and a fast rise. Beyond the general observations that a plateau is usually a two-phase coexistence region $(1,2,18)$ and that the fast-rising region resembles that of the "solid" phases of LMs on water $(1,2,18)$, no definite structural conclusions can be drawn on the basis of the isotherm alone.

Fresnel-normalized XR curves, measured at $A$ values indicated in Fig. 1, are shown in Fig. 2A (circles) with their model fits (lines). The surface-normal electron density profiles derived from the fits are plotted in Fig. 2B. The bare $\mathrm{Hg}$ (inset, curve 1) exhibits a surface-induced layering peak at $q_{z} \approx 2.2 \AA^{-1}$ observed previously for bare (20-22) and thiol-covered (12) Hg surfaces. The good fit to the measured XR (curve 2) reveals a uniform LM of thickness $d=4.8 \AA$ and electron density $\rho=0.30 \mathrm{e} / \AA^{3}$. These values agree closely with the interchain distance (19) and the electron density (23) of close-packed al-

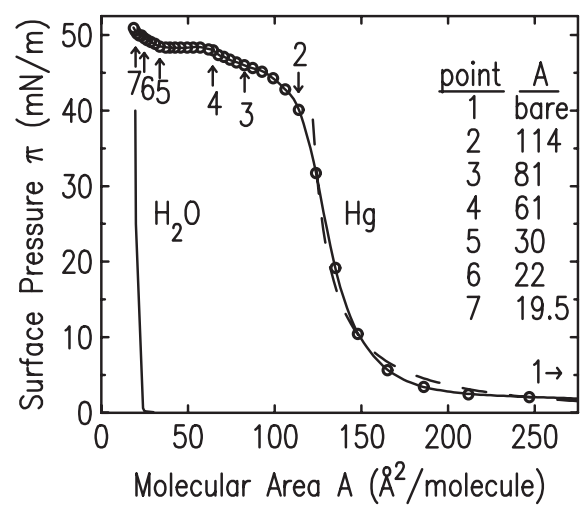

Fig. 1. Surface pressure $(\pi) /$ molecular area (A) isotherms of a LM of stearic acid on $\mathrm{Hg}$ (solid line with circles) and on water (solid line without circles) at room temperature. X-ray reflectivity was measured at the numbered points. The dashed line is an isotherm of a hard-sphere gas. The molecular area $A$ was varied by stepwise deposition of a chloroform solution of stearic acid onto the fixed-area trough $(6.5 \mathrm{~cm}$ by $17.5 \mathrm{~cm}$ ), enclosed in a He-filled box and mounted on a diffractometer. The surface pressure was measured by a film balance with a $\mathrm{Hg}$-amalgamated platinum plate. 


\section{R E P O R T S}

kyl chains. This and the isotherm strongly support the conclusion that for $A \geq 110$ $\AA^{2} /$ molecule, the LM is a monolayer of surface-parallel molecules. The detection by XR of such thin films, $d \leq 5 \AA$, is possible only on liquid metal surfaces, where the high surface tension, $\gamma \approx 500 \mathrm{mN} / \mathrm{m}$ for $\mathrm{Hg}$, results in a very low surface roughness, $\sigma \leq 1 \AA$. The larger $\sigma=3$ to $4 \AA$ of an aqueous surface $(24,25)(\gamma \approx 72 \mathrm{mN} / \mathrm{m})$ precludes reliable XR studies of LMs less than $\sim 15 \AA$ thick.

The XR curves 3 and 4 , at $A=81$ and 61 $\AA^{2} /$ molecule, reveal the successive growth of a second layer of surface-parallel molecules on top of the first layer. The fits yield a full coverage for the first layer and densities
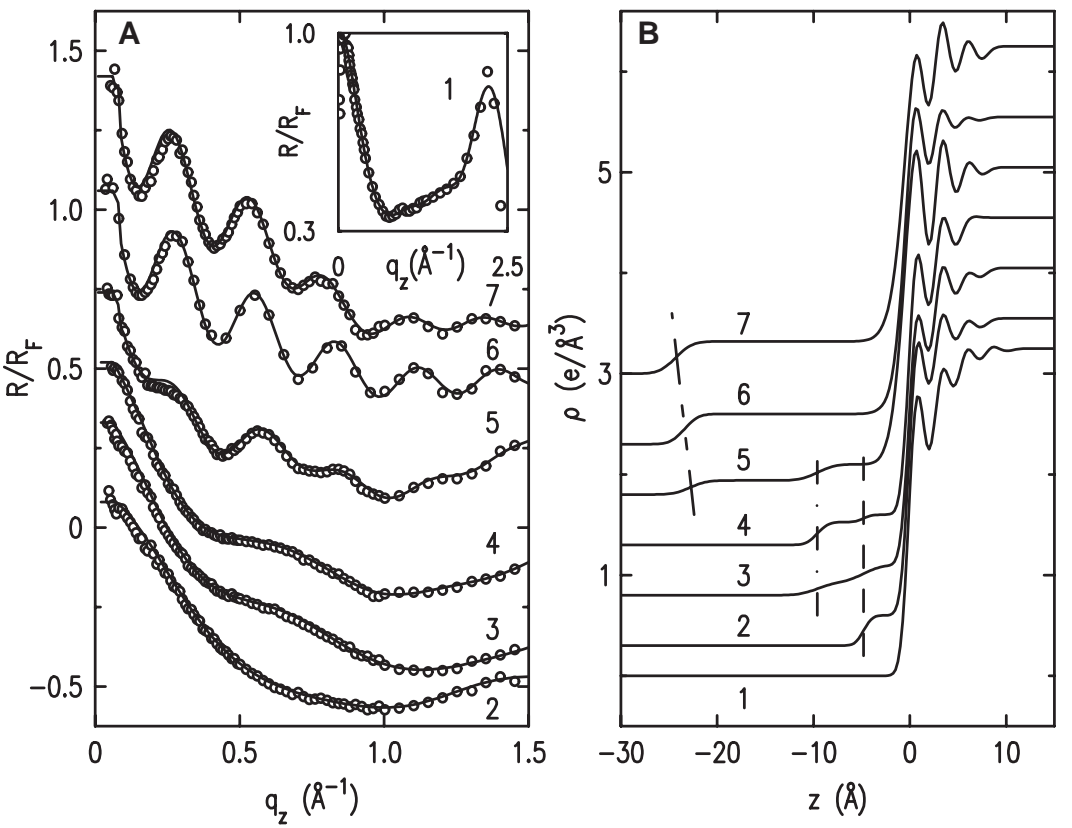

Fig. 2. X-ray reflectivity at the numbered points in Fig. 1. (A) The measured and Fresnel-normalized reflectivities, $R / R_{\mathrm{F}}\left(q_{\mathrm{z}}\right)$ (circles), and slab-model fits (lines). The model includes one (single-layer) or two (double-layer) slabs for the flat-lying phases (points 1 to 4), a variable fraction coverage by a flat-lying double layer (two slabs) and a standing-up monolayer (one slab) in the coexistence region (point 5), and a two-slab model (head and tail groups) for the standing-up monolayer (points 6 and 7). (B) The surface-normal electron density profiles, $\rho(z)$, derived from the fits. The positive $z$ axis points into the liquid, with $\mathrm{z}=0$ denoting the $\mathrm{Hg}$ surface. The dash, dash-dot, and dash-dot-dot lines denote the tops of the flat-lying single and double layers and the standing-up monolayer, respectively. Curves 2 to 7 are shifted for clarity in (A) by $-0.87,-0.64,-0.45,-0.22,0.1$, and 0.45 , and in (B) by $0.3,0.8,1.3,1.8,2.3$, and 3 , respectively. $X$-ray measurements were done at the Harvard/BNL Liquid Spectrometer, beamline X22B, National Synchrotron Light Source, at a wavelength of $1.5560 \AA$.

Table 1. Grazing incidence $x$-ray diffraction results for the standing-up phases. $q_{\|, p}$ and $q_{z, p}$, GID and BR peak positions, respectively; $\theta$, molecular tilt; $a$ and $b$, unit cell dimensions; and $\delta$, lattice distortion. The isotherm's average $(A), x$-ray derived $O_{v}$ and LS phase $\left(A_{x}\right)$, and chain-normal $(A \perp)$ areas per molecule are also listed.

\begin{tabular}{lcccccccccc}
\hline $\begin{array}{c}A \\
\left(\AA^{2} / \mathrm{mol}\right)\end{array}$ & $\begin{array}{c}q_{\|, \rho}(11) \\
\left(\AA^{-1}\right)\end{array}$ & $\begin{array}{c}q_{\|, \rho}(02) \\
\left(\AA^{-1}\right)\end{array}$ & $\begin{array}{c}q_{z, \rho, \rho}(02) \\
\left(\AA^{-1}\right)\end{array}$ & $\begin{array}{c}q_{z,, \rho}(11) \\
\left(\AA^{-1}\right)\end{array}$ & $a(\AA)$ & $b(\AA)$ & $\theta$ & $\begin{array}{c}A_{x} \\
\left(\AA^{2} / \mathrm{mol} .\right)\end{array}$ & $\begin{array}{c}A \perp \\
\left(\AA^{2} / \mathrm{mol} .\right)\end{array}$ & $\delta$ \\
\hline 30 & 1.377 & 1.451 & 0.78 & 0.39 & 4.919 & 9.126 & 30 & 22.5 & 19.5 & -0.070 \\
28 & 1.373 & 1.449 & 0.79 & 0.42 & 4.924 & 9.152 & 30 & 22.5 & 19.4 & -0.072 \\
25 & 1.426 & 1.472 & 0.60 & 0.30 & 4.879 & 8.812 & 23 & 21.5 & 19.8 & -0.042 \\
23 & 1.459 & 1.487 & 0.50 & 0.27 & 4.849 & 8.613 & 19 & 20.9 & 19.7 & -0.025 \\
20 & 1.507 & 1.507 & 0 & 0 & 4.814 & 8.339 & 0 & 20.1 & 20.1 & 0 \\
19.5 & 1.525 & 1.525 & 0 & 0 & 4.758 & 8.240 & 0 & 19.6 & 19.6 & 0 \\
\hline
\end{tabular}

double-layer phase in this $A$ range, over the "crumpled molecule" phase, suggested on the basis of the isotherm alone (9).

The conclusion is that for $A \geq 52 \AA^{2 /}$ molecule, the structure is dominated by the strong chain-subphase attraction, which induces surface-parallel molecular alignment in the LM. The attraction is, however, insufficient to induce a third layer of surface-parallel molecules. Upon increasing the coverage further, the molecules start standing up.

The XR on the plateau, e.g., curve 5 at $A=30 \AA^{2} /$ molecule, shows short-period Kiessig-like fringes, which could be fitted only by assuming a two-phase coexistence between a double layer of surface-parallel molecules and a monolayer of standing-up molecules. The fits, which were done by keeping the double-layer's $d$ and $\rho$ fixed at the $A=52 \AA^{2} /$ molecule values, show for curve 5 that $47 \%$ of the surface area is covered by standing-up molecules, which includes $69 \%$ of the molecules. The fitted thickness of the monolayer, $d=22.3 \AA$, is less than the extended molecular length (19), $24.4 \AA$, indicating an average $\sim 24^{\circ}$ molecular tilt (26) from the surface normal. Decreasing $A$ from 35 to $25 \AA^{2} /$ molecule increases the area covered by the standing-up monolayer from 24 to $77 \%$, with $d$ increasing from 22.1 to $23.2 \AA$, which indicates an accompanying decrease in the molecular tilt from $\sim 25^{\circ}$ to $\sim 18^{\circ}$.

The coexistence range ends at $A \approx 23$ $\AA^{2} /$ molecule. For lower values of $A$ the XR can be modeled by a single monolayer of standing-up molecules, with the carboxyl heads residing at the $\mathrm{Hg}$ surface. For curves 6 and 7 , at $A=22$ and $19.5 \AA^{2} /$ molecule, a layer thickness of 23.3 and $24.3 \AA$ is found, corresponding to estimated tilts of $\sim 17^{\circ}$ and $\leq 5^{\circ}$ (the XR detectable limit). The XR-derived structure in this range is very similar to that of LMs of stearic $(1,8)$ and arachidic (1, 27) $\left(\mathrm{C}_{20} \mathrm{H}_{40} \mathrm{O}_{2}\right)$ acids on water at similar values of $A$, demonstrating the dominance of chain-chain interactions over chain-subphase attraction in this region.

GID and BR patterns measured for the highdensity standing-up phases at several coverages are shown in Fig. 3, with peaks indexed in a body-centered rectangular lattice (1). Table 1 lists the GID-derived data for these phases. For $A \geq 23 \AA^{2} /$ molecule, the molecules tilt toward next-nearest neighbors (NNNs), as evidenced by the BR's peak positions, $q_{z, p}(02) \approx 2 \times q_{z, p}$ $(11) \neq 0$, and their $1: 2$ intensity ratio, resulting from the twofold degeneracy of the (11) peak and the nondegeneracy of the (02) peak (1). The $\mathrm{NNN}$ tilt and the undistorted hexagonal packing in the chain-normal plane identify this as the Overbeck-Möbius phase $(1,28), \mathrm{O}_{\mathrm{v}}$. The monolayer-plane distortions of the packing from a perfect hexagon $(1), \delta=(8 / 3)\left[q_{\|, p}(11)\right.$ $\left.-q_{\|, p}(02)\right] /\left[q_{\|, p}(11)+q_{\|, p}(02)\right]$, are found to be 
REPORTS

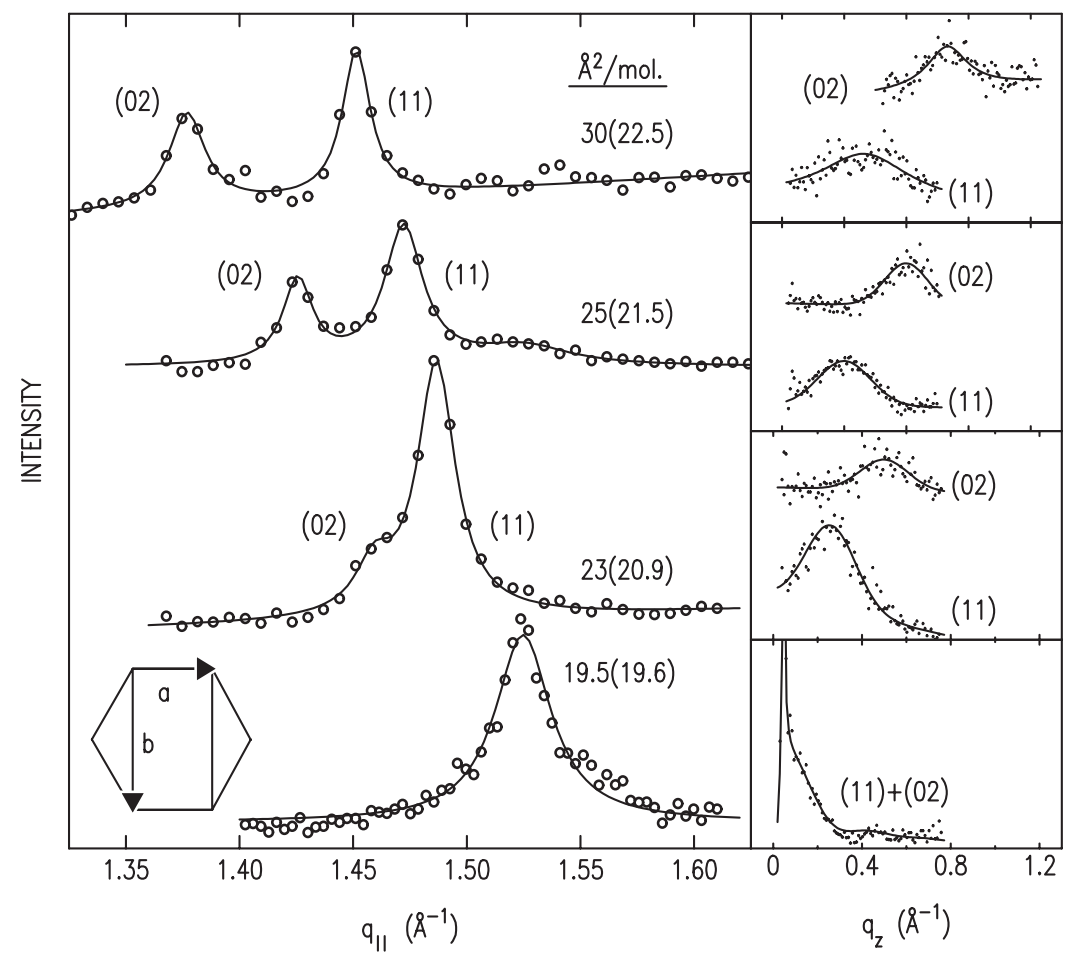

Fig. 3. Measured (circles and points) and model-fitted (lines) GID (left) and BR (right) patterns for the tilted and untilted phases of $A \leq 30 \AA^{2} /$ molecule. The peaks are indexed in the body-centered rectangular unit cell shown at lower left. The molecular areas in parentheses were derived from the $\mathrm{x}$-ray data. Values derived from these measurements are listed in Table 1.

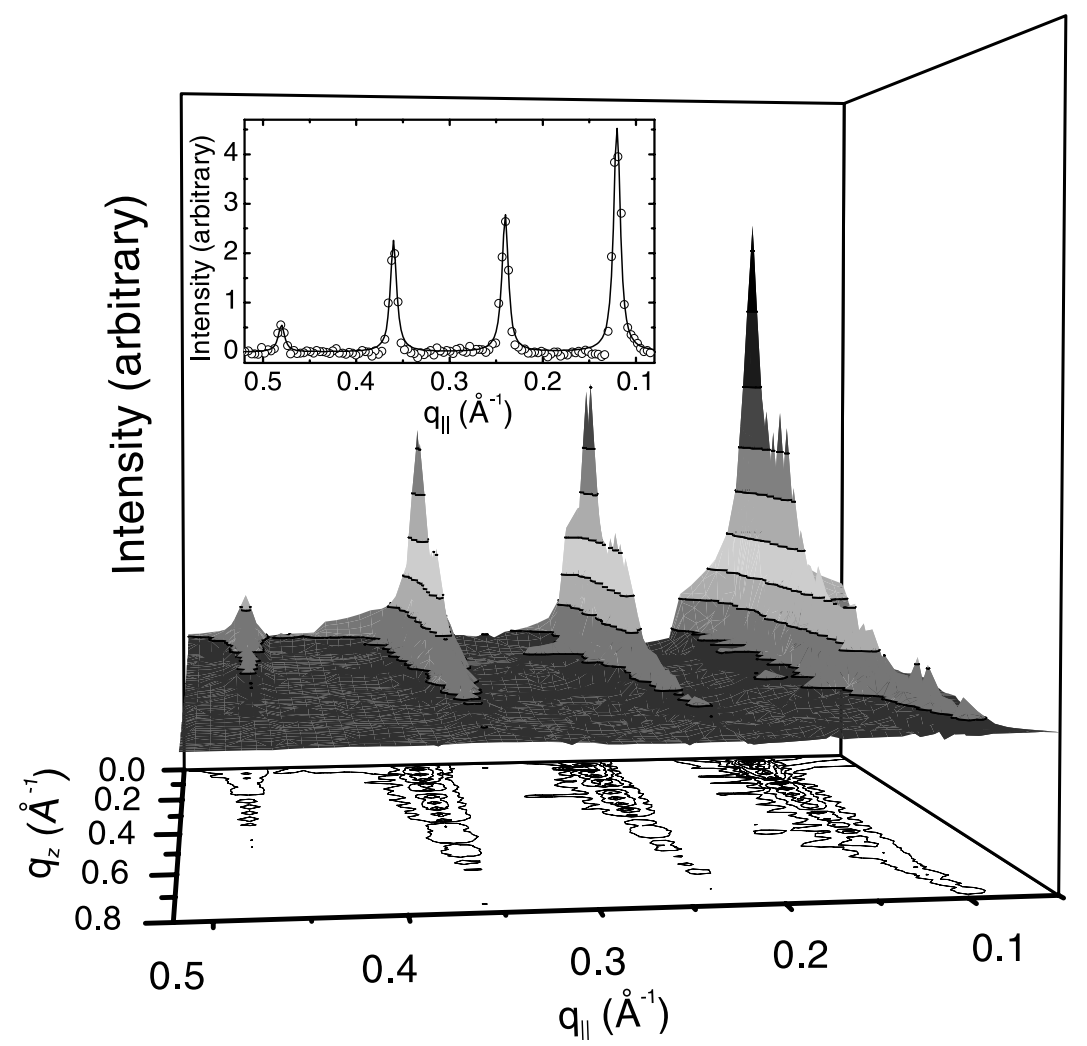

Fig. 4. The measured $\left(q_{\|}, q_{z}\right)$ surface diffraction pattern for the flat-lying double-layer phase of stearic acid on $\mathrm{Hg}$. A contour plot of the BRs is shown below the gray scale-coded pattern. The peak positions in the GID scan $\left(q_{z}\right.$-integrated intensity versus $\left.q_{\|}\right)$in the inset are multiples of $q_{\|}=0.120$ $\AA^{-1}$, indicating a repeat distance corresponding to molecular dimers. linear in $\sin ^{2} \theta=q_{z, p}^{2}(02) /\left[q_{\|, p}^{2}(02)+q_{z, p}^{2}(02)\right]$, where $\theta$ is the tilt angle. The intersection of this line with the origin proves (1) that the lattice distortion is induced by the tilt, and not by a reorientation of the molecular backbone planes. For the highest $\pi$ (lowest $A$ ), the single GID peak and the BR's $q_{z, p}=0$ prove a hexagonal packing of untilted molecules, i.e., a super liquid (LS) phase (1). The same phases, $\mathrm{O}_{\mathrm{v}}$ and LS, were also found, with very similar unit cell dimensions and tilts, for stearic acid LMs on water $(8)$ at high $\pi$, where they show hexatic order, and disordered backbone plane orientations $(1,29)$. The lower symmetry phases found on water $(8,29)$, some exhibiting $\mathrm{NN}$ tilts and almost all having herringbone order for the backbone planes, were not detected here. Perhaps this is because the surface pressures at which these phases occur in water-supported LMs are much lower than the surface pressure $\pi \geq 48 \mathrm{mN} / \mathrm{m}$ at which the standing-up phases are observed in our Hg-supported LMs. Some of these lower symmetry phases may appear in $\mathrm{Hg}$-supported LMs at temperatures below the room temperature at which the present study was performed.

The two low-coverage phases of flat-lying molecules exhibit several low- $q_{\|}$GID peaks, all multiples of $q_{\|}=0.120 \AA^{-1}$ (Fig. 4, inset). This indicates a well-ordered structure with a repeat distance $d_{\|}=52.4 \AA, \sim 6 \%$ larger than that of bulk stearic acid (30), $d_{\text {bulk }}=49.3 \AA$. All bulk crystalline polymorphs of stearic acid consist of dimers, hydrogen bonded at their center by the carboxylic head groups of the two molecules (19). The close correspondence between $d_{\|}$and $d_{\text {bulk }}$ implies that our low-coverage phases consist of in-plane ordered uniform-width stripes of flat-lying dimers, oriented along the stripe's width. The BR results, shown in Fig. 4 as a 3D pattern and as contour plots, strongly support this conclusion. The published repeat distances of Langmuir-Blodgett multilayers of metal stearates range from $49.9 \AA$ [Mn stearate (31)] to $50.6 \AA[\mathrm{Pb}$ stearate (32)], all within $\sim 1 \AA$ of $d_{\text {bulk }}$, and considerably smaller than $d_{\|}=52.4$ $\AA$ of our flat-lying phases. Thus, repeat-distance considerations and/or the BR fits can neither support nor rule out a possible incorporation of $\mathrm{Hg}$ atoms into the flat-lying dimer's center, as is found in self-assembled multilayers of surface-normal thiol molecules (33). The origin of the $\sim 3 \AA$ difference between the repeat distances of flat-lying (here) and surfacenormal (bulk) dimer phases is unclear and merits further study. Finally, no GID peaks were found for the flat-lying phases at $1.2 \leq q_{\|} \leq 1.4$ $\AA^{-1}$, which would correspond to an order along the stripes, normal to the dimers' long axis. Therefore, we conclude that the GID results suggest a 2D smectic-like structure for the flatlying phases, i.e., a 1D longitudinal positional order within a quasi-2D layer.

The results above indicate that the strong surface-chain attraction dominates the structure 
of the Hg-supported LMs, either directly, by inducing the surface-parallel single- and double-layer phases, or indirectly, by imposing a high $\pi$-threshold on the standing-up phases and thus eliminating the low- $\pi$ standing-up phases observed in water-supported LMs. Hg-supported LMs, in which the film thickness and inplane structure can be tuned in situ by varying the surface pressure, and in particular the phases revealed here, may prove more advantageous for studying nanosized electronic devices than the fixed-structure thiol self-assembled monolayers being used at present $(34,35)$. They should provide new insight into the nature of the molecular-level interactions between organic and metallic molecules. Understanding such interactions is a central aim of two very important emerging fields: nanofabrication (36) and biometallic interfacing (37-39). They may also help to resolve fundamental controversies such as whether conformational disorder inhibits or promotes charge transfer across a monolayer in $\mathrm{Hg}$-organics heterostructured devices (40-42). Our findings should hopefully lead to studies of LMs on additional subphases, further elucidating the subphase's important role in determining the LM's structure, and to studies of LMs of more complex systems on $\mathrm{Hg}$ subphases, such as fullerenes, nanotubes, and metallic nanoparticles, of much current interest to both basic and applied science.

References and Notes

1. V. M. Kaganer, H. Möhwald, P. Dutta, Rev. Mod. Phys. 71, 779 (1999).

2. I. Kuzmenko et al., Chem. Rev. 101, 1659 (2001).

3. A. Pockels, Nature 43, 437 (1891).

4. J. Jortner, M. Ratner, Eds., Molecular Electronics (Blackwell, Oxford, UK, 1997).

5. S. Grayer, Wolf et al., Nature 328, 63 (1987).

6. K. Kjaer, J. Als-Nielsen, C. A. Helm, L. A. Laxhuber, H. Möhwald, Phys. Rev. Lett. 58, 2224 (1987).

7. P. Dutta, J. B. Peng, B. Lin, J. B. Ketterson, M. Prakash, Phys. Rev. Lett. 58, 2228 (1987).

8. I. R. Peterson, G. Brezesinski, B. Struth, E. Scalas, J. Phys. Chem. B 102, 9437 (1998).

9. T. Smith, Adv. Colloid Interface Sci. 3, 161 (1972).

10. S. W. Barton, B. N. Thomas, E. B. Flom, F. Novak, S. A. Rice, Langmuir 4, 233 (1988).

11. B. Harzallah, L. Bosio, R. Cortes, N. Errafii, J. Chim. Phys. (France) 93, 1202 (1996).

12. O. M. Magnussen et al., Nature 384, 250 (1996).

13. R. C. Weast, M. J. Astle, W. H. Beyer, Eds., Handbook of Chemistry and Physics (CRC, Boca Raton, FL, ed. 66, 1986), pp. F174-F184.

14. M. Deutsch, B. M. Ocko, in Encyclopedia of Applied Physics, G. L. Trigg, Ed. (VCH, New York, 1998), vol. 23, p. 479.

15. J. Daillant, A. Gibaud, Eds., X-Ray and Neutron Reflectivity: Principles and Applications (Springer, Berlin, 1999).

16. J. Als-Nielsen et al., Phys. Rep. 246, 252 (1994).

17. J. Daillant, M. Alba, Rep. Prog. Phys. 63, 1725 (2000).

18. G. L. Gaines, Insoluble Monolayers at Liquid-Gas Interfaces (Wiley, New York, 1966).

19. D. M. Small, The Physical Chemistry of Lipids (Plenum, New York, 1986).

20. O. M. Magnussen et al., Phys. Rev. Lett. 74, 4444 (1995).

21. The suprasurface "beach" observed there is replaced here by an equivalent subsurface depletion region (26).

22. E. Dimasi, H. Tostmann, B. M. Ocko, P. S. Pershan, M. Deutsch, Phys. Rev. B 58, 13419 (1998).
23. X. Z. Wu, E. B. Sirota, S. K. Sinha, B. M. Ocko, M. Deutsch, Phys. Rev. Lett. 70, 958 (1993).

24. A. Braslau et al., Phys. Rev. Lett. 54, 114 (1985).

25. C. Fradin et al., Nature 403, 871 (2000).

26. The tilts derived from the $x$-ray reflectivity have measurement and fit uncertainties of $\sim 4^{\circ}$ to $5^{\circ}$.

27. B. Lin, M. C. Shih, T. M. Bohanon, G. E. Ice, P. Dutta, Phys. Rev. Lett. 65, 191 (1990).

28. G. A. Overbeck, D. Möbius, J. Phys. Chem. 97, 7999 (1993).

29. V. M. Kaganer, E. B. Loginov, Phys. Rev. Lett. 71, 2599 (1993).

30. M. Popescu, T. Gutberlet, M. Kastowsky, P. J. Koch, H. Bradaczek, Thin Solid Films 304, 323 (1997).

31. S. Hazra, A. Gibaud, A. Desert, V. Gacem, N. Cowlam, Physica B 283, 45 (2000).

32. P. Ganguly, D. V. Paranjape, S. K. Chaudhari, J. Phys. Chem. 97, 11965 (1993).

33. M. Deutsch, O. M. Magnussen, B. M. Ocko, M. J. Regan, P. S. Pershan, in Thin Films: Self-Assembled Monolayers of Thiols, A. Ulman, Ed. (Academic Press, San Diego, CA, 1998), pp. 179-203.

34. M. A. Rampi, O. J. A. Schueller, G. M. Whitesides, Appl. Phys. Lett. 72, 1781 (1998).

35. K. Slowinski, H. K. Y. Fong, M. Majda, J. Am. Chem. Soc. 121, 7257 (1999).
36. A. Hatzor, P. S. Weiss, Science 291, 1119 (2001).

37. B. Kasemo, Surf. Sci. 500, 656 (2002).

38. E. Braun, Y. Eichen, U. Sivan, G. Bar-Yoseph, Nature 391, 775 (1998).

39. Y. Maeda, H. Tabata, T. Kawai, Appl. Phys. Lett. 79, 1181 (2001).

40. C. Boulas, J.V. Davidovits, F. Rondelez, D. Vuillaume, Phys. Rev. Lett. 76, 4797 (1996).

41. A. Haran, D. H. Waldeck, R. Naaman, E. Moons, D. Cahen, Science 263, 948 (1994).

42. K. Slowinski, M. Majda, J. Electroanal. Chem. 491, 139 (2000).

43. Support to P.S.P. by the U.S. Department of Energy (grant DE-FG02-88-ER45379) and the National Science Foundation (grant DMR-01-124936) and to M.D. by the United States-Israel Binational Science Foundation, Jerusalem, is gratefully acknowledged. We thank D. Vaknin (Ames) for generous advice and drawings of the trough, and Y. Rabin and I. Kanter for a critical reading of the manuscript. Brookhaven National Laboratory is supported by the U.S. Department of Energy (contract DE-AC02-98CH10886).

11 September 2002; accepted 16 October 2002

\title{
Simulation of Subduction Zone Seismicity by Dehydration of Serpentine
}

\author{
David P. Dobson, ${ }^{1,2 *}$ Philip G. Meredith, ${ }^{2}$ Stephen A. Boon ${ }^{2}$
}

\begin{abstract}
We measured acoustic emission energy during antigorite dehydration in a multianvil press from 1.5 to 8.5 gigapascals and $300^{\circ}$ to $900^{\circ} \mathrm{C}$. There was a strong acoustic emission signal on dehydration, and analysis of recovered samples revealed brittle deformation features associated with high pore-fluid pressures. These results demonstrate that intermediate depth (50 to $200 \mathrm{ki}-$ lometers) seismicity can be generated by dehydration reactions in the subducting slab.
\end{abstract}

The origin of intermediate- and deep- (50 to $600 \mathrm{~km}$ ) focus earthquakes within subducting oceanic slabs remains enigmatic. At these depths, rocks should deform by plastic flow rather than by the sudden brittle deformation that is prevalent in the shallow crust. Subducting oceanic lithosphere undergoes changes in mineralogy with increasing pressure and temperature, and these reactions have been invoked to explain deep earthquakes (1-4). Dehydration of the subducting slab and migration of fluids generates magma below back-arcs $(5,6)$, but it is currently unclear whether these phase transformations can generate seismicity under the pressure-temperature $(P-T)$ conditions of the subducting slab.

We performed acoustic emission (AE) studies of antigorite dehydration between 1.5 and $8.5 \mathrm{GPa}$ under conditions pertinent to subduction. AEs are high-frequency elastic

'Bayerisches Geoinstitut, Universität Bayreuth, D-95440 Bayreuth, Germany. ${ }^{2}$ Department of Earth Sciences, University College London WC1E 6BT, UK.

*To whom correspondence should be addressed. Email: d.dobson@ucl.ac.uk waves produced by rapid strain in solids and are analogous to seismic waves within Earth. Antigorite is the major hydrous phase in serpentinized peridotites and can be used as a proxy for hydrous phases in the slab. AE signals just above the dehydration temperature demonstrate that elastic strain waves associated with rapid deformation are a characteristic feature of dehydration under these conditions.

Experiments were performed in a $1000-$ metric-ton split-cylinder multianvil press (7). Cylinders $3 \mathrm{~mm}$ in diameter and $4 \mathrm{~mm}$ in length were cored from a natural Erzgebirge garnet serpentinite, and garnet-free cores were chosen as samples. These consisted of 90 to $95 \%$ antigorite $\left[\mathrm{Mg}_{41.6} \mathrm{Fe}^{2+}{ }_{1.22}\right.$ $\left.\mathrm{Al}_{0.34} \mathrm{Fe}^{3+}{ }_{3.17} \mathrm{Si}_{34} \mathrm{O}_{85}(\mathrm{OH})_{62}\right]$ with minor chromium-diopside, olivine, and oxides. Samples were placed centrally in a stepped $\mathrm{LaCrO}_{3}$ furnace in the high-pressure cell. Acoustic waveguides were placed betweenthe sample and the anvils. We used either polycrystalline corundum or iron waveguides. Corundum has a low attenuation, maximizing the observed AE signal, but gen- 